\title{
Robust Economic Model Predictive Control of Drinking Water Transport Networks Using Zonotopes
}

\author{
Khoury Boutrous $^{(\bowtie)}$, Fatiha Nejjari, and Vicenç Puig \\ Advanced Control Systems Group, Technical University of Catalonia (UPC), \\ Rambla Sant Nebridi 22, 08222 Terrassa, Spain \\ \{boutrous.khoury, fatiha.nejjari, vicenc.puig\}@upc.edu
}

\begin{abstract}
A robust economic Model Predictive Control (EMPC) approach is presented in this paper for the control of a Drinking Water Network (DWN) albeit the presence of uncertainties in the forecasted demands required for the predictive control design. The uncertain forecasted demand on the nominal MPC has the possibility of rendering the optimization process infeasible or degrade the controller performance. In this paper, the uncertainty on demand is considered unknown but bounded in a zonotopic set. Based on this uncertainty description, a robust MPC is formulated to ensure robust constraint satisfaction, performance and stability of the MPC for DWN to meet user requirements whilst ensuring lower operational cost for water utility operators.
\end{abstract}

Keywords: Model predictive control $\cdot$ Economic control • Robustness $\cdot$ Drinking water network $\cdot$ Zonotopes

\section{Introduction}

Model predictive control (MPC) stands out as the predominant modern controller used in industry, mainly due to its inherent ability to ensure closed-loop stability and constraint satisfaction whilst satisfying a cost function. Recently, the concept of incorporating directly an economic stage cost of industrial processes in a MPC design termed economic MPC has garnered interest with a plethora of applications as proposed in $[11,12]$ showing promising results. This procedure involves an update of the generic cost function which normally involves tracking a set-point to one which explicitly involves economic terms such as energy and cost of production, enabling an improvement during transients and the ability to manipulate control variables to satisfy various economic requirements. The problem of stability in economic MPC has been extensively studied in [13]. As the name suggests, there exits economic entities (such as e.g. cost. price or demand) that serves as exogenous variables in the design of economic MPC. These variables are undoubtedly subject to stochastic variations which requires further control design considerations for a suitable operation. For example, in 
the design of an economic MPC with variable demand as done in [10], a forecast of demand to enable future predictions of states in the MPC optimization loop is required. But forecasted demand as a variable is subject to human behaviour which can be described as uncertain at best. Therefore, there is the need to ensure that controllers are built robust to design variations, which are inevitable in real life situations. Methods of stochastic MPC [5], the Min-Max Robust formulation [9], the tree-based method [8] and other proposed concepts have been successfully applied to problems of uncertainties in internal or exogenous parameters in MPC optimization formulations, but most of these methods yields computationally demanding problems. [6] provides a comprehensive overview of robust MPC, highlighting recent trends, limitations and proposed future research directions.

The prime objective of a DWN operation is to ensure that demand nodes in a network are served with quality water at desired pressures whilst minimising operational cost. EMPC in the area of water supply has been extensively studied. In [4], the large number of actuators (i.e. decision variables) in the DWN was parameterized to minimize the number of decision variables in the optimization loop ensuring a faster run-time. [10] designs a two level control scheme, where the upper level controls the complete system via a nonlinear MPC scheme and the lower level, a pump scheduling scheme through mixed integer programming, but these proposed schemes fails to account for uncertainties in the forecasted demand which may affect controller performance. Stochastic MPC for uncertainties at demand nodes of a DWN has been studied by [5] showing promising results of maintaining tractability and performance of the system subject to uncertainties but provides a somewhat computational expensive solution.

In this paper, a robust EMPC is designed for a DWN, specifically the case of the Barcelona Drinking Water Network, considering uncertainties in forecasted demand. The variations in demand are considered unknown but bounded, and are described to vary in a zonotopic set. Zonotopic sets show desirable characteristics of less complexity, flexibility and reliable computation of linear transformation and Minkowski sums compared to other geometric counterparts such as interval, ellipsoidal sets e.t.c [3]. The associated effect of the uncertainty in the demand on the states and inputs are described offline, taking advantage of the affine dependence of these variables on the demand as given in the model description of the DWN. Hence, a reachable set is constructed offline. The offline description of these sets provides a similar formulation of an optimization problem to the nominal controller, therefore offering similar optimization complexity. However, it must be noted that even though a robust MPC is achieved after this procedure, there is a certain degree of robustness for some magnitude of disturbance beyond which the optimization problem ceases to be feasible. The constraint formulations are then updated to ensure robust constraints satisfaction as well as a local LQR feedback control designed to mitigate the effect of the uncertainty on the predicted states as done in [2]. The rest of this paper is structured as follows: a mathematical preliminary to the concept of zonotopes will be given in Sect. 1. Then, a description of a linear DWN model to be used for the controller design is presented in Sect. 2. Section 3 presents the design of 
the robust MPC scheme and finally results and conclusions after application of the developed scheme is discussed in Sect. 4.

\subsection{Mathematical Preliminaries}

Definition 1. The Minkowski sum of two sets, $\mathcal{X}$ and $\mathcal{Y}$ is defined as $\mathcal{X} \oplus \mathcal{Y}=$ $\{x+y: x \in \mathcal{X}, y \in \mathcal{Y}\}$.

Definition 2. A $m$-zonotope, $\mathcal{Z} \in \mathbb{R}^{n}$ is defined as the linear image of a $m$ dimensional hypercube, where $m$ is the order of the zonotope and subsequently satisfies $(m \geq n)$. Given a vector $\mathbf{P} \in \mathbb{R}^{n}$ and a matrix $\mathbf{H} \in \mathbb{R}^{n \times m}$, a $m$-zonotopic set $\mathcal{Z}$, can be described as:

$$
\mathcal{Z}=\mathbf{P} \oplus \mathbf{H} B^{m}=\left\{x \in \mathbb{R}^{n} \mid x=p+H z, z \in B^{m}\right\} .
$$

Which defines the Minkowski sum of the $m$-segments defined by $m$-columns of matrix $\mathbb{H} \in \mathbb{R}^{n}$. Given $p$, the center of the zonotope $\mathcal{Z}$ and $\mathbf{H}=\left[h_{1}, h_{i}, \ldots . h_{n}\right]$ $\forall h_{i} \in \mathbb{R}^{n}$ as the generators of the zonotope. Properties governing computations of zonotopes are given as follows:

Property 1. The Minkowski sum of two zonotopes $\mathcal{Z}_{1}=\mathbf{P}_{1} \oplus \mathbf{H}_{1} B^{n_{1}}$ and $\mathcal{Z}_{2}=\mathbf{P}_{2} \oplus \mathbf{H}_{2} B^{n_{2}}$ is a zonotope defined by $\mathcal{Z}=\left(\mathbf{P}_{1}+\mathbf{P}_{2}\right) \oplus\left[\mathbf{H}_{1}, \mathbf{H}_{2}\right] B^{n_{1}+n_{2}}$.

Property 2. The image of a zonotope $\mathcal{Z}=\mathbf{P} \oplus \mathbf{H} B^{n}$ by linear mapping matrix $K$ can be calculated as $K \mathcal{Z}=K \mathbf{P} \oplus K \mathbf{H} B^{n}$.

\section{DWN Control-Oriented Model}

DWN comprises of a complex network of hydraulic elements that convey water from supply to demand [1]. These elements work interactively to satisfy demands at desired pressure and of good water quality during transportation. The network can thus be categorized into the supply, treatment, transport and distribution sub-levels. Active hydraulic elements (pumps and valves) control flow and/or pressure in specific paths in the network, interactively working with passive elements such as pipes and tanks which act as transport and storage elements respectively to satisfy the broader network requirements in terms of meeting demand as well as ensuring water supply by storing adequate water reserves in the network's tanks. There have been several attempts in literature to model DWNs which captures key dynamics at different levels of the drinking water network architecture. Through graph theory, some works consider flow directions of water at network nodes as well as interactions at the tanks [1] leading to a simple flow-based model description of the network, whilst other studies [5] consider both flow and pressure specifically taking into account the interactions when flow and hydraulic head equations are considered in the modelling process. Considering only the transport sub-level, the flow-based model offers an easier option to work with, largely due to its linearity but fails to captures 
key pressure dynamics which is important to present a complete mathematical behaviour of the network. Inclusion of pressure in the DWN dynamics introduces non-linearity from the pressure-flow affine equality equation into the optimization problem's constraints formulation resulting in a non-convex problem. Some works have been successful in designing nonlinear MPC [14] for the control of these nonlinear models, whilst works such as [15] considers nonlinear constraint relaxation to produce a set of linear inequality constraints for a linear Economic EMPC formulation. Despite its complexity, the nonlinear pressure-flow model offers a more realistic case to work with. The purpose of this paper is to only illustrate the ability of a set-based method (zonotope) of robust EMPC to ensure suitable controller performance under demand uncertainties, hence the comparatively less complex flow based model will be used. [7] presents a flow-based model on the Barcelona water network. Basic relationships between elements considering mass balance at tanks and equilibrium of flow directions at nodes, give rise to a discrete time dynamics as follows:

$$
\begin{gathered}
x_{k+1}=A x_{k}+B_{u} u_{k}+B_{d} d_{k}, \\
0=E_{u} u_{k}+E_{d} d_{k} .
\end{gathered}
$$

$x_{k} \in \mathbb{R}_{+}^{n_{x}}$ is the vector of system states, denoting tank volumes at each time instant $k . u_{k} \in \mathbb{R}^{n_{u}}$ denotes the manipulated input from actuators affecting changes in states in combination with the non-negative model disturbance $d_{k} \in \mathbb{R}_{+}{ }^{n_{d}}$, that represents the consumer demand. A, $B_{u}, B_{d}, E_{u}$ and $E_{d}$ are time-invariant matrices of suitable dimensions. From Eq. (3), it can be inferred that the control variable $u$ does not take its value from $\mathbb{R}^{n_{u}}$ but in a linear variety. This inference enables an affine parameterisation of the control variables in terms of a minimum set of disturbance, mapping the control problem to a space with a smaller decision vector and with less computational burden due to the elimination of the equality constraint, (3) [10].

Assumption 1: Considering that there are more variables than algebraic equations (i.e. $n_{q}<n_{u}$ ), the matrix $E_{u}$ in (3) has a maximal rank. Therefore, it can be expressed in a reduced staggered form using the Gauss-Jordan elimination.

From Assumption 1, the control variable is parameterized such that:

$$
u_{k}=\tilde{P} \tilde{M}_{1} \hat{u}_{k}+\tilde{P} \tilde{M}_{2} d_{k}
$$

The model can be represented as a difference Eq. (5) by replacing (4) into Eq. (2), given as:

$$
x_{k+1}=A x_{k}+\hat{B} \hat{u}_{k}+\hat{B}_{d} d_{k}
$$

where $\hat{B}=B \tilde{P} \tilde{M}_{1}$ and $\hat{B}_{d}=B \tilde{P} \tilde{M}_{2}+B_{d}$.

For an in-depth understanding on how the control variables are parameterized, reader is referred to [10]. 


\section{Problem Formulation}

The problem of designing a robust MPC controller must be such that the designed controller satisfies the tenets of robust stability, robust constraint satisfaction and robust performance for all realization of a system $\Sigma=f(u, x, d)$, albeit any variations of function variables. Assuming demand uncertainty in the system, the effects of these uncertainties on the exogenous demand variable $d_{k}$, $\Delta d_{k} \subseteq \delta d$ results in a subsequent variation in state $\Delta x_{k} \subseteq \delta X$ and input variables $\Delta u_{k} \subseteq \delta U$ as described in the affine relationships of Eqs. (2) and (3). Model variables, $(d, x, u)$ can subsequently be decomposed into an uncertainty free and uncertain dependent component, with the latter involving a realization of variables at each time instant from bounded uncertainty sets, $(\delta d, \delta U, \delta X)$. Thus, the state and input uncertainty sets $(\delta X, \delta U)$ described as a zonotope are generated from the known zonotopic bounded set of the demand variation $\delta d$ with the aid of the algebraic difference Eqs. (2)-(3).

Assumption 2: The states $x$ and demands $d$ are considered observable at each time instant $k$ and the pair $(A, \hat{B})$ is controllable.

$\delta d$ is bounded using a zonotope that can be formulated from a symmetric interval set considering bounded demand uncertainty such that $\Delta d_{i} \in\left[-\Delta d_{i}, \Delta d_{i}\right]$, where $i$ denotes a particular demand node. The set $\delta d$ can therefore be represented in a zonotopic form as:

$$
\delta d_{k}=0 \oplus H_{d} z_{d} .
$$

Where 0 is a column vector of dimension $n_{d}$ ( $n_{d}$ is the number of demand nodes), considered as the center the zonotope and $H_{d}$ is a diagonal matrix of the generators represented as the bounds of variations at each demand node $i$ : $z_{d} \in B^{n_{d}} ; B=\left[\begin{array}{ll}-1 & 1\end{array}\right]$. Considering that $\tilde{x}, \tilde{\hat{u}}$ and $\tilde{d}$ are the real dynamic states, inputs and demand respectively, which takes into account the uncertainty effects, appropriate decomposition of model variables is therefore given as: $\tilde{x}=x+\Delta x$, $\tilde{\hat{u}}=\hat{u}+\Delta \hat{u}$ and $\tilde{d}=d+\Delta d$.

The DWN model considering uncertainty in the demand variable is therefore given as from (2) and (3) as:

$$
\begin{gathered}
\tilde{x}_{k+1}=A \tilde{x}_{k}+\hat{B} \tilde{\hat{u}}_{k}+\hat{B}_{d} \tilde{d}_{k}, \\
0=E_{u} \tilde{\hat{u}}_{k}+E_{d} \tilde{d}_{k} .
\end{gathered}
$$

Nominal states $x \in \mathbb{R}_{+}{ }^{n_{x}}$ and inputs $\hat{u} \in \mathbb{R}_{+}{ }^{n_{u}}$ are considered bounded in a compact polyhedron $\mathbb{U}$ and $\mathbb{X}$ respectively, containing the origin in their interiors, with $\hat{u} \subseteq \mathbb{U}$ and $x \subseteq \mathbb{X}$. In the presence of uncertainty, it is desirable to generate a tube of trajectories, meaning a sequence of robust invariant reachable sets such that per every transition of states and inputs of the nominal system, the resultant states and inputs after effect of uncertainty remains in a closed and bounded set. A robust invariant tube, $\tilde{X}_{k}=\left\{\tilde{X}_{0}, \tilde{X}_{1}, \ldots, \tilde{X}_{N}\right\}, \forall \tilde{X}_{k}=x_{k} \oplus \delta X_{k}$ and subsequent control tube $\tilde{U}_{k}=\left\{\tilde{U}_{0}, \tilde{U}_{1}, \ldots, \tilde{U}_{N}\right\}, \forall \tilde{U}_{k}=\hat{u}_{k} \oplus \delta U_{k}$, is constructed either online or offline, with the offline procedure offering lesser computational 
burden. $x_{k}$ and $\hat{u}_{k}$ are centers of the respective propagated state and control invariant tubes. The mismatch between nominal predicted states and real states resulting from uncertainties are mitigated by a local feedback controller $K$, in this case an LQR controller such that the selection of this feedback gain $K$ satisfies a system with the assumption that $\tilde{d}_{k}=0$

$$
\Delta x_{k+1}=(A+\hat{B} K) \Delta x_{k}
$$

with $\Delta x_{k} \subseteq \delta X_{k}$. The local controller ensures that the deviation of the system dynamics in the closed loop with $(A+\hat{B} K)$ is asymptotically stable. The primary aim is to have an optimal control problem, which keeps trajectories around the neighbourhood of the nominal optimal trajectory in the presence of uncertainties, satisfying the cost function under process constraints.

Assumption 3: Considering that $(A+\hat{B} K)$ is strictly stable and $\tilde{x}=x+\Delta x$, such that the uncertain dynamic part $\Delta x_{k+i+1}=(A+\hat{B} K) \Delta x_{k+i}+\hat{B}_{d} \Delta d_{k+i}$. If $(A+\hat{B} K) \delta X \oplus \hat{B}_{d} \delta d \subseteq \delta X$, then it can be assumed that the transition of states from one time instant to another depends on the dynamics of the centers $x_{k+i+1}=A x_{k+1}+\hat{B} \hat{u}_{k+1}+\hat{B}_{d} d_{k+1}$.

\subsection{Offline Computation of Zonotopic Reachable Sets}

The feedback gain $K$ is computed and kept constant at each time instant $k$ throughout the prediction horizon of the MPC controller to minimize the deviation of the perturbed state as stated before. The following cost function is utilized for the design of an optimal local controller for state error minimization

$$
J_{\infty}=\sum_{i=0}^{\infty}\left(\tilde{x}_{k}-x_{k}\right)^{T} Q\left(\tilde{x}_{k}-x_{k}\right)+\sum_{i=0}^{\infty}\left(\tilde{\hat{u}}_{k}\right)^{T} R\left(\tilde{\hat{u}}_{k}\right) .
$$

Where $Q$ and $R$ are positive definite matrices of appropriate dimensions, $\tilde{x}$ is the real state at time $k$ from the real plant and $\tilde{\hat{u}}$, the actual inputs, with $x_{k}$ as the nominal state prediction from the MPC at time instant $k$. From (7), the real state, $\tilde{x}_{k}$ (i.e. $\tilde{x}_{k}=x_{k}+\Delta x_{k}$ ) under conditions of uncertainty as stated before can be decomposed into:

Certain component: $\left\{x_{k+1}=A x_{k}+\hat{B} \hat{u}_{k}+\hat{B}_{d} d_{k}\right.$.

Uncertain component: $\left\{\Delta x_{k+1}=(A+\hat{B} K) \Delta x_{k}+\hat{B}_{d} \Delta d_{k}\right.$.

where $\Delta \hat{u}=K \Delta x$, from the local LQR controller. From the uncertain component, a corresponding $N \in \mathbb{Z}_{>0}$ length of tube is computed, where $N$ is the selected prediction horizon of the MPC controller. Therefore, realization of the deviation $\Delta x$ from the set $\delta X$ assuming that initial deviation, $\Delta x_{0}=0$ can be described as:

$$
\delta X_{k+j} \subseteq \bigoplus_{i=1}^{j}(A+\hat{B} K)^{j-i} \hat{B}_{d} \delta d
$$


Given that $\delta d=0 \oplus H_{d} B^{n d}$, and from properties 1 and 2 of the zonotope, it therefore follows that:

$$
\begin{gathered}
\delta X_{k+j} \subseteq 0 \oplus \Psi_{1, j} B^{n_{d}}, \\
\Psi_{1, j}=\bigoplus_{i=1}^{j}(A+\hat{B} K)^{j-i} \hat{B}_{d} H_{d} .
\end{gathered}
$$

From (4) in the control parameterization as discussed in Sect. 2, the auxiliary control variable $\tilde{\hat{u}}$ at time instant $k$ can be described as:

$$
\tilde{\hat{u}}_{k}=\hat{u}_{k}+K \Delta x_{k},
$$

where $\hat{u}_{k}$ is the certain control variable at time $k$ with associated uncertainty, $\Delta \hat{u}_{k} . K$ is the local controller gain calculated from (10). From Eq. (4), and under decomposition into certain and uncertain parts, considering that the actual control variable $\tilde{\hat{u}}_{k}$ is a value from a set i.e. $\tilde{\hat{u}} \in \delta U$, the uncertain control set is given as:

$$
\delta U_{k+j} \subseteq \tilde{P} \tilde{M}_{1} K \delta X_{k+j} \oplus \tilde{P} \tilde{M}_{2} \delta d,
$$

Equation (15) is obtained considering the control parameterization (4). The sequence of cross-sections of the control tube can therefore be described in a zonotopic form as

$$
\delta U_{k+j} \subseteq 0 \oplus\left[\tilde{P} \tilde{M}_{1} K \Psi_{1, j}, \tilde{P} \tilde{M}_{2} H_{d}\right] B^{2 n_{d}} .
$$

\subsection{Terminal State Constraint Set}

For robust stability and recursive feasibility, a terminal constraint set is formulated. A minimal terminal robust positive invariant set $\delta X_{f}$ is constructed as an outer approximation of the exact equilibrium state set

$$
\tilde{X}_{*}=\bigoplus_{j=0}^{\infty}(A+\hat{B} K)^{j} \hat{B}_{d} \delta d
$$

where $\tilde{X}_{*} \subseteq \delta X_{f}$. Given that $(A+\hat{B} K)=\hat{A}$ and $\hat{B}_{d} \delta d=\mathcal{W}$ and under the assumption that $\hat{A}$ is strictly stable, with $\mathcal{W} \subseteq \delta d$. An outer set approximation is assumed if there exist a certain $k \in \mathbb{Z}_{>0}$ such that, $(\hat{A})^{k} \mathcal{W} \subseteq \alpha \mathcal{W}, \forall \alpha=[0,1)$. The infinite Minkowski sum of sets (17) under strict stability conditions ensures that convergence is guaranteed. Considering the infinite Minkowski sum,

$$
\bigoplus_{j=0}^{\infty}(\hat{A})^{j} \mathcal{W}=\bigoplus_{j=0}^{k-1}(\hat{A})^{j} \mathcal{W} \oplus \bigoplus_{j=k}^{2 k-1}(\hat{A})^{j} \mathcal{W} \oplus \bigoplus_{j=2 k}^{3 k-1}(\hat{A})^{j} \mathcal{W} \oplus \ldots
$$

Equation (18) can be simplified to achieve the condition $(\hat{A})^{k} \mathcal{W} \subseteq \alpha \mathcal{W}$ as follows:

$$
\bigoplus_{j=0}^{\infty}(\hat{A})^{j} \mathcal{W}=\bigoplus_{j=0}^{k-1}(\hat{A})^{j} \mathcal{W} \oplus \bigoplus_{j=0}^{k-1}(\hat{A})^{j}(\hat{A})^{k} \mathcal{W} \oplus \bigoplus_{j=0}^{k-1}(\hat{A})^{j}(\hat{A})^{2 k} \mathcal{W} \oplus \ldots
$$


From $(\hat{A})^{k} \mathcal{W} \subseteq \alpha \mathcal{W}$, it can be stated that $(\hat{A})^{n k} \mathcal{W} \subseteq \alpha^{n} \mathcal{W}$. Taking $\bigoplus_{j=0}^{k-1}(\hat{A})^{j} \mathcal{W}$ to be equal to $\Pi$ and considering Eq. (18), $\tilde{X}_{*}$ can be approximated from a truncation of (18) as:

$$
\delta X_{f}=\left(1+\alpha+\alpha^{2}+\ldots \ldots\right) \Pi,
$$

which results in an approximated set as given below:

$$
\delta X_{f} \subseteq(1-\alpha)^{-1} \Pi,
$$

the set in a zonotope form, as described by the properties in Sect.1.1 is given as:

$$
\begin{gathered}
\delta X_{f} \subseteq 0 \oplus(1-\alpha)^{-1} \Psi_{2, j} B^{n_{d}} . \\
\Psi_{2, j}=\bigoplus_{j=0}^{k-1}(\hat{A})^{j} \hat{B}_{d} H_{d} .
\end{gathered}
$$

Thus, the size of the set is dependent on the design parameter $\alpha$. The terminal state of the real system, $\tilde{x}_{n}$ under uncertainty, belongs to an invariant set, $\tilde{X}_{f} \forall \tilde{x}_{n} \subseteq \tilde{X}_{f}$. The uncertain terminal state can henceforth be expressed in a decomposed form as:

$$
x_{n} \oplus \delta X_{f} \subseteq \tilde{X}_{f} .
$$

The constructed sequence of uncertain zonotopic sets will then be used in the sequel for the design of the robust EMPC by considering only alterations in the constraints.

\subsection{Robust EMPC Formulation}

From Assumption 2, the optimization problem for the economic cost minimization involves the minimization of the centers of the tube, the cost function therefore involves deterministic variables. According to [5], for the nominal MPC synthesis of a DWN, the objective function $\mathcal{L}(k, \hat{u}, x)$, involves three terms:

- To account for variable electricity at the pumps and production costs, the following term is included

$$
\mathcal{J}_{E}(k)=\left(\alpha_{1}+\alpha_{2}\right)^{T} \hat{u}(k),
$$

where $\alpha_{1}$ is a fixed cost related to the water production and $\alpha_{2}$ is the time varying electricity cost.

- A penalty equal to the sum of the squares of the deviation of the volume in each tank, $i$ from a predefined safety threshold

$$
\mathcal{J}_{s}(k)=\sum_{i=1}^{T}\left\|\varphi_{i}(k)\right\|^{2},
$$

where $\varphi_{i}(\mathrm{k})$ denotes the deviation of the stored volume in tank, $i$ of $T$ tanks below the desired minimum volume. 
- A penalty on the square of the flow variations in the actuators, i.e., the slew rate

$$
\mathcal{J}_{\triangle U}(k)=\|\triangle \hat{u}(k)\|^{2},
$$

where $\triangle \hat{u}(k)=\hat{u}(k)-\hat{u}(k-1)$.

The resultant objective function is given as follows:

$$
\therefore \mathcal{L}(k, \hat{u}, x)=\lambda_{1} \mathcal{J}_{s}(k)+\lambda_{2} \mathcal{J}_{\triangle \hat{U}}(k)+\lambda_{3} \mathcal{J}_{E}(k) .
$$

Where $\lambda_{1}, \lambda_{2}$ and $\lambda_{3}$ are design weights for each objective criterion.

The proposed robust approach only involves an update of the constraints considering $\mathbb{U} \oplus \delta U \subseteq \mathcal{U}, \mathbb{X} \oplus \delta X \subseteq \mathcal{X}$ and $X_{f} \oplus \delta X_{f} \subseteq \tilde{X}_{f}$

$$
\begin{gathered}
\min _{\hat{\mathbf{u}}(k), \mathbf{x}(k)} \sum_{i=0}^{N-1} \mathcal{L}(k, \hat{u}, x) \\
\text { s.t. } \quad x_{k+i+1 \mid k}=A x_{k+i \mid k}+\hat{B} \hat{u}_{k+i \mid k}+\hat{B}_{d} d_{K+i \mid k}, \\
\hat{u}_{k+i \mid k} \subseteq \mathcal{U} \ominus \delta U, \\
x_{k+i+1 \mid k} \subseteq \mathcal{X} \ominus \delta X, \\
x_{k+N \mid k} \subseteq X_{f}, \\
\tilde{x}_{k}-x_{k} \subseteq \delta X .
\end{gathered}
$$

Considering that the optimization problem is feasible, i.e., there exists a non-empty solution given by the optimal sequence of control inputs $\left(\hat{u}^{*}(0), \hat{u}^{*}(1), \ldots \hat{u}^{*}(N-1)\right)$, where $N$ is the prediction horizon. From the principles of receding horizon, only the first control action $\hat{u}^{*}(0 \mid k)$ of the sequence $N$ values obtained from the solution of the MPC optimization problem is applied to the plant.

$$
\hat{u}(k)=\hat{u}^{*}(0 \mid k),
$$

disregarding the rest of control actions. At the next time instant $k$, the optimization problem is solved again using the current measurements of states and disturbances, with the most recent forecast of the latter over the next future horizon. From the control parameterization (4), the control input to the plant at every time instant $k$ is given by

$$
u^{*}(0 \mid k)=\tilde{P} \tilde{M}_{1} \hat{u}^{*}(0 \mid k)+\tilde{P} \tilde{M}_{2} \tilde{d}(k)+K \Delta x(k) .
$$

Where $\tilde{d}(k)$ is the forecasted demand including uncertainty measured at time instant $k$. 


\section{Results}

The proposed controller is applied to the aggregate model of the Barcelona drinking water network, which consists of 17 tanks, 61 control variables (26 pumps and 35 valves), 11 nodes and 25 demand points. As discussed in the modeling of the DWN, through control parametrization, the decision variables are reduced to 50 from 61 to ease computational burden and assist in formula ting the uncertainty sets. The weights $\lambda$, in the multi-objective problem, (27) are selected such that more priority is placed on the minimization of the economic cost against the other competing objective criteria of maintaining proper levels of safety volumes and control action smoothness. $\lambda_{1}, \lambda_{2}$ and $\lambda_{3}$ are chosen as 100, 10 and 0.1 respectively. Real demand data at different demand nodes are subsequently used as the forecasted model disturbance.

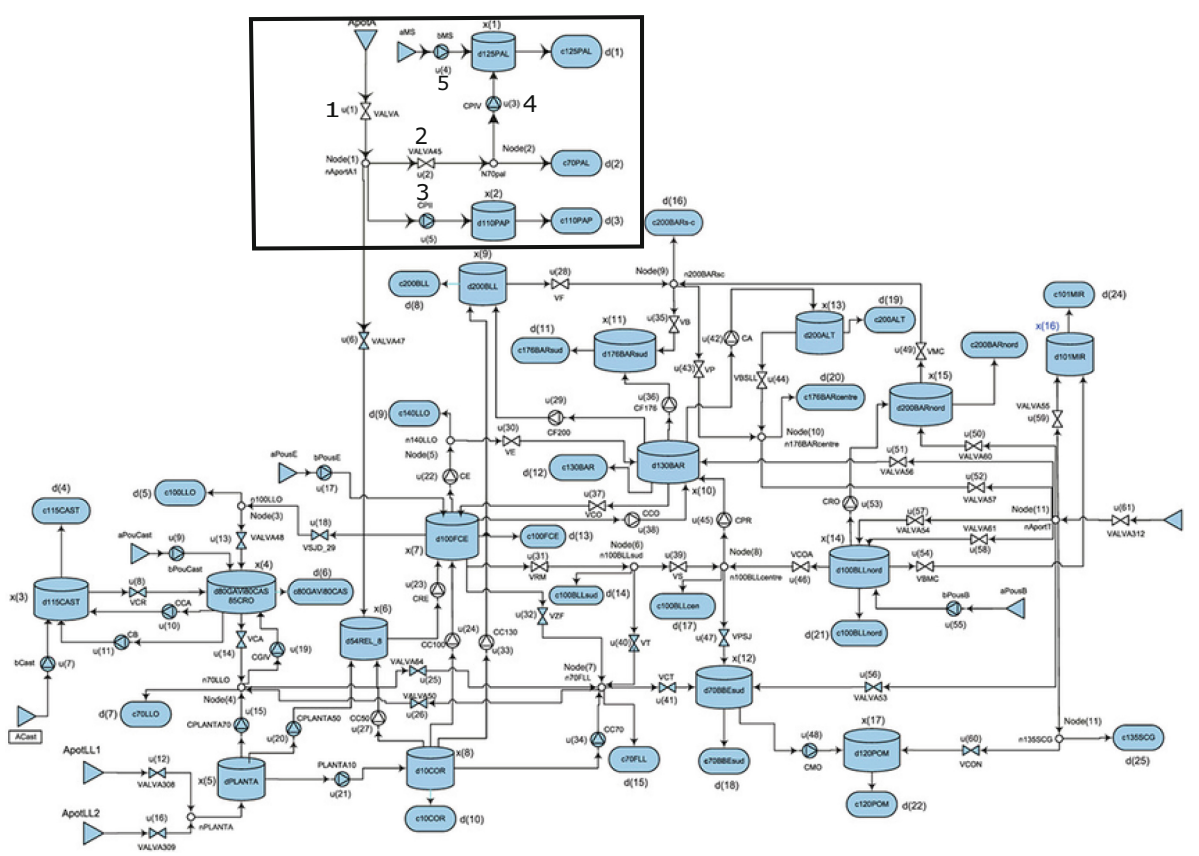

Fig. 1. Aggregate model of the Barcelona water network

\subsection{Simulated Results}

In the simulations, the additive demand uncertainty is taken as a variation between $\pm 5 \%$ of maximum forecasted demand which is illustrated in Fig. 2 for one demand node C70PAL as shown in Fig. 1, from which the corresponding 
uncertain zonotopic set is formulated. The prediction horizon is $24 \mathrm{~h}$, with a sampling time of $1 \mathrm{~h}$. The MPC optimization problem (28) is solved with CPLEX ${ }^{\circledR}$ QP solver, with Yalmip and Matlab ${ }^{\mathrm{B}}$ R2017b (64 bits) using a PC with an Intel core i7 with $8 \mathrm{~GB}$ of RAM. To demonstrate the capabilities of the proposed controller, it is compared with a nominal EMPC controller of similar parameters but ignoring the demand uncertainties.

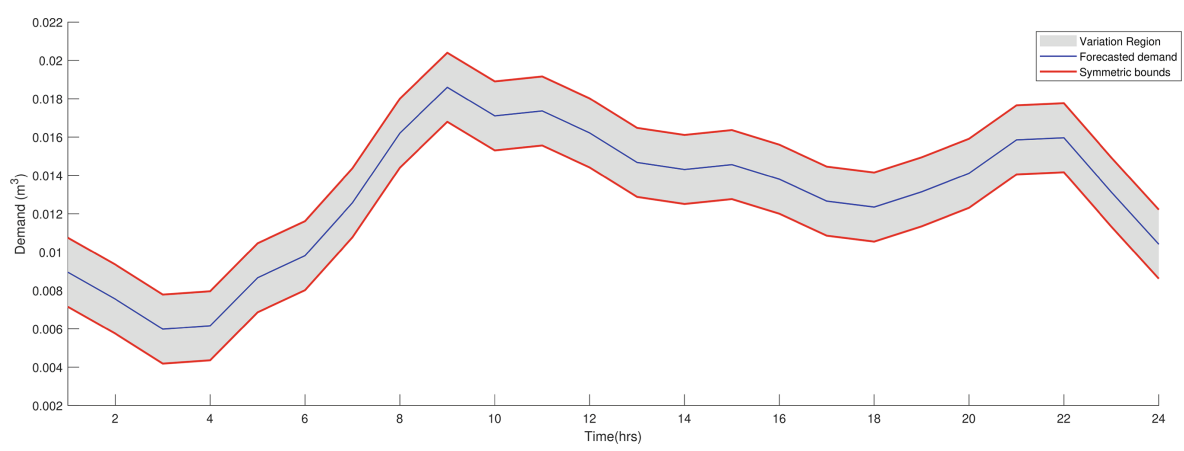

Fig. 2. Bounds on forecasted demand at C70PAL for $24 \mathrm{~h}$

The dynamics of some selected elements, Tanks - $(254 R E L, d 110 P A P \&$ d125PAL) and Actuators - $\left(V A L M A^{1}, V A L M A 45^{2}, C P I I^{3}, C P I V^{4} \& B M S^{5}\right)$ of the aggregate model with the actuators enumerated in order as listed in Fig. 1 are shown for purposes of comparison (Figs. 3 and 4) between the proposed robust and nominal EMPC controllers. The robust controller is augmented with a local controller to ensure that the deviation between nominal state and perturbed state is minimized, which can be tuned accordingly with $Q$ and $R$ of the LQR controller. From Fig. 3, the robust EMPC enables convergence to a neighbourhood of the nominal state in the presence of demand uncertainties, maintaining an almost similar behaviour of the network tank level as the nominal controller. The main control objective of the predictive controller is to reduce operational cost due to the actuator effort from the linear cost $\mathcal{J}_{E}(k)$. Summation of consumer demand occurs at some nodes, therefore there is an aggregation of additive uncertainties, $\Delta d$ on actuators that effect flow when connected to nodes that has multiple demand points propagated to. From Fig. 4, the Robust EMPC shows similar control efforts as the nominal controller, especially with pumps not connected directly to demand points as the magnitude of uncertainty is comparatively less. For actuators connected directly to some nodes, for example, in the case of actuator $V A L M A^{1}$, a marginally higher deviation is realised, this can be attributed to accumulated demand variations at node NOP18 propagated to the actuator. Deviations are also due to the demand variations assumed measured for control inputs at each sampling time arising from the control parameterization (30). Also infeasible solutions were realised in the case of the nominal EMPC controller under the same uncertainty propagation. 


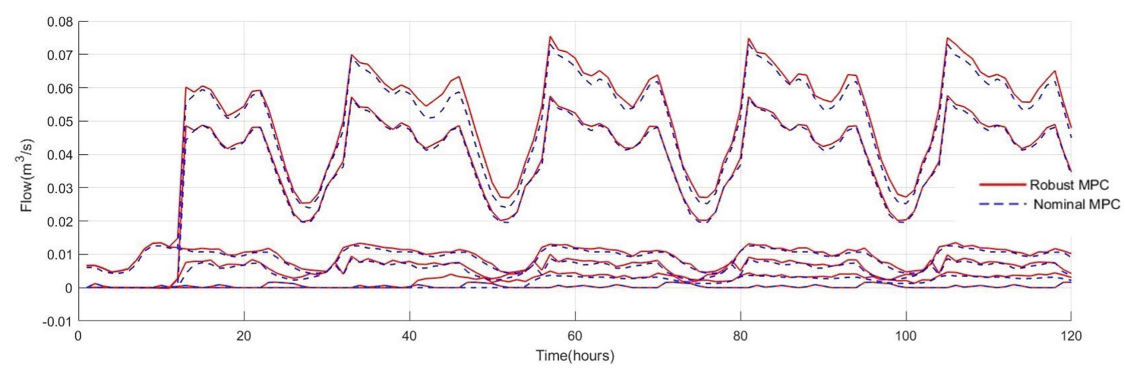

Fig. 3. Comparison of selected actuator dynamics for Robust and Nominal MPC

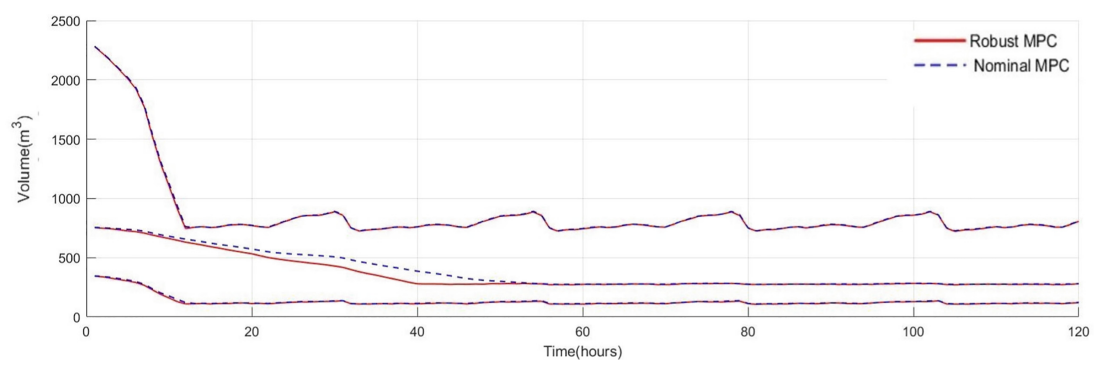

Fig. 4. Level dynamics of tanks for Robust and Nominal MPC

\section{Conclusions}

This paper seeks to address the problem of model uncertainty associated with the design of MPC, specifically in relation to demand uncertainties in a drinking water network. Uncertainties on demand are considered unknown but bounded in a zonotope. Then, a robust EMPC is designed based on this description, which shows similar behaviour when compared to a nominal EMPC without demand uncertainties satisfying intended control objectives and most importantly the robust EMPC preserves stability and feasibility. The optimization problem of the nominal EMPC is observed to be infeasible when subjected to the same magnitude of variation as the robust EMPC. As a future work, it is planned to compared the proposed approach with the chance-constraints approach introduced in [10] on DWNs.

\section{References}

1. Grosso, J., Ocampo-Martinez, C., Puig, V., Limon, D., Pereira, M.: Economic MPC for the management of drinking water networks. In: 2014 European Control Conference (ECC), pp. 2739-2744 (2014)

2. Pereira, M., Muñoz, D.P., Limon, D.: Robust economic model predictive control of a community micro-grid. In: 2016 IEEE 55th Conference on Decision and Control (CDC), pp. 2739-2744 (2017) 
3. Le, V., Stoica, M., Alamo, T.: Zonotopes; From Guaranteed State Estimation to Control. Wiley, Hoboken (2013)

4. Cembrano, G., Quevedo, J., Puig, V., Pérez, R., Figueras, J., Escaler, I., Ramón, G., Barnet, G., Rodríguez, P., Casas, M.: A generic tool for real-time operational predictive optimal control of water networks. J. Int. Assoc. Water Pollut. Res. 64(2), 448-459 (2011)

5. Wang, Y., Puig, V., Cembrano, G.: Non-linear economic model predictive control of water distribution networks. J. Process Control 55(6), 23-34 (2017)

6. Bemporad, A., Morari, M.: Robust model predictive control: a survey. In: Garulli, A., Tesi, A. (eds.) Robustness in Identification and Control, vol. 245, pp. 207-226. Springer, London (1999)

7. Puig, V., Escobet, T., Sarrate, R., Quevedo, J.: Fault diagnosis and fault tolerant control in critical infrastructure systems. In: Kyriakides, E., Polycarpou, M. (eds.) Intelligent Monitoring, Control, and Security of Critical Infrastructure Systems. Studies in Computational Intelligence, vol. 565, pp. 263-299. Springer, Heidelberg (2010)

8. Velarde, P., Maestre, J.M., Ocampo-Martinez, C., Bordons, C.: Application of robust model predictive control to a renewable hydrogen-based microgrid. In: 2016 European Control Conference (ECC), pp. 1209-1214 (2016)

9. Löfberg, J.: Min-max approaches to robust model predictive control. Linköping Studies in Science and Technology. Dissertations (2003)

10. Grosso, J.M., Velarde, P., Ocampo-Martinez, C., Maestre, J.M., Puig, V.: Stochastic model predictive control approaches applied to drinking water networks. Optim. Control Appl. Methods 38(4), 541-558 (2017)

11. Jain, T., Yamé, J.J.: IEEE Trans. Sustain. Energy 4(4), 1696-1704 (2019)

12. Durand, H., Christofides, P.D.: Economic model predictive control for nonlinear processes incorporating actuator magnitude and rate of change constraints. In: 2016 American Control Conference (ACC), pp. 5068-5074 (2016)

13. Angeli, D., Amrit, R., Rawlings, J.B.: On average performance and stability of economic model predictive control. IEEE Trans. Autom. Control 57(7), 1615-1626 (2012)

14. Wang, Y., Puig, V., Cembrano, G.: Non-linear economic model predictive control of water distribution networks. J. Process Control 56(8), 23-34 (2017)

15. Wang, Y., Puig, V., Cembrano, G.: Economic model predictive control with nonlinear constraint relaxation for the operational management of water distribution networks. Energies 11(4), 991-999 (2018) 